\title{
Renal transplant complication: Ureteral obstruction
}

\author{
Steve Roumpf, Julie L. Welch and Greg Zahn
}

Clinical Emergency Medicine, Department of Emergency Medicine, Indiana University School of Medicine, Indianapolis, IN, USA

Keywords: Renal transplant, Kidney transplant, Inguinal hernia

65 yo male with a kidney transplant presented for nausea, abdominal pain, and decreased urine output. Physical exam revealed RLQ abdominal tenderness and a non-reducible right inguinal hernia. Abdominal/Pelvis CT showed obstruction of the right ureter within an inguinal hernia with hydronephrosis of the transplanted kidney (Images 1 and 2). Labs revealed acute renal failure (creatinine $9.32 \mathrm{mg} / \mathrm{dl})$, hyperkalemia $(6.0 \mathrm{mmol} / \mathrm{l})$, and gap acidosis. Nephrology was consulted for emergent hemodialysis. General Surgery and Urology were consulted for the incarcerated ureter, obstruction, and hernia. Interventional Radiology placed a right percutaneous nephrostomy tube to relieve the renal obstruction (Image 3). After stabilization, he had an inguinal hernia repair with reduction of his transplant ureter.

This is the author's manuscript of the work published in final edited form as: 


\section{References}

1. Hart A., et al: OPTN/SRTR 2016 Annual data report: kidney. Am J Transplant 2018; 18: pp. 18-113

2. KDIGO clinical practice guidelines for acute kidney injury. Nephron Clin Pract 2012; 120: pp. 179-184

3. Cheungpasitporn W.: Incidence of kidney stones in kidney transplant recipients: A systematic review and meta-analysis. World J Transplant 2016; 6: pp. 790-797 


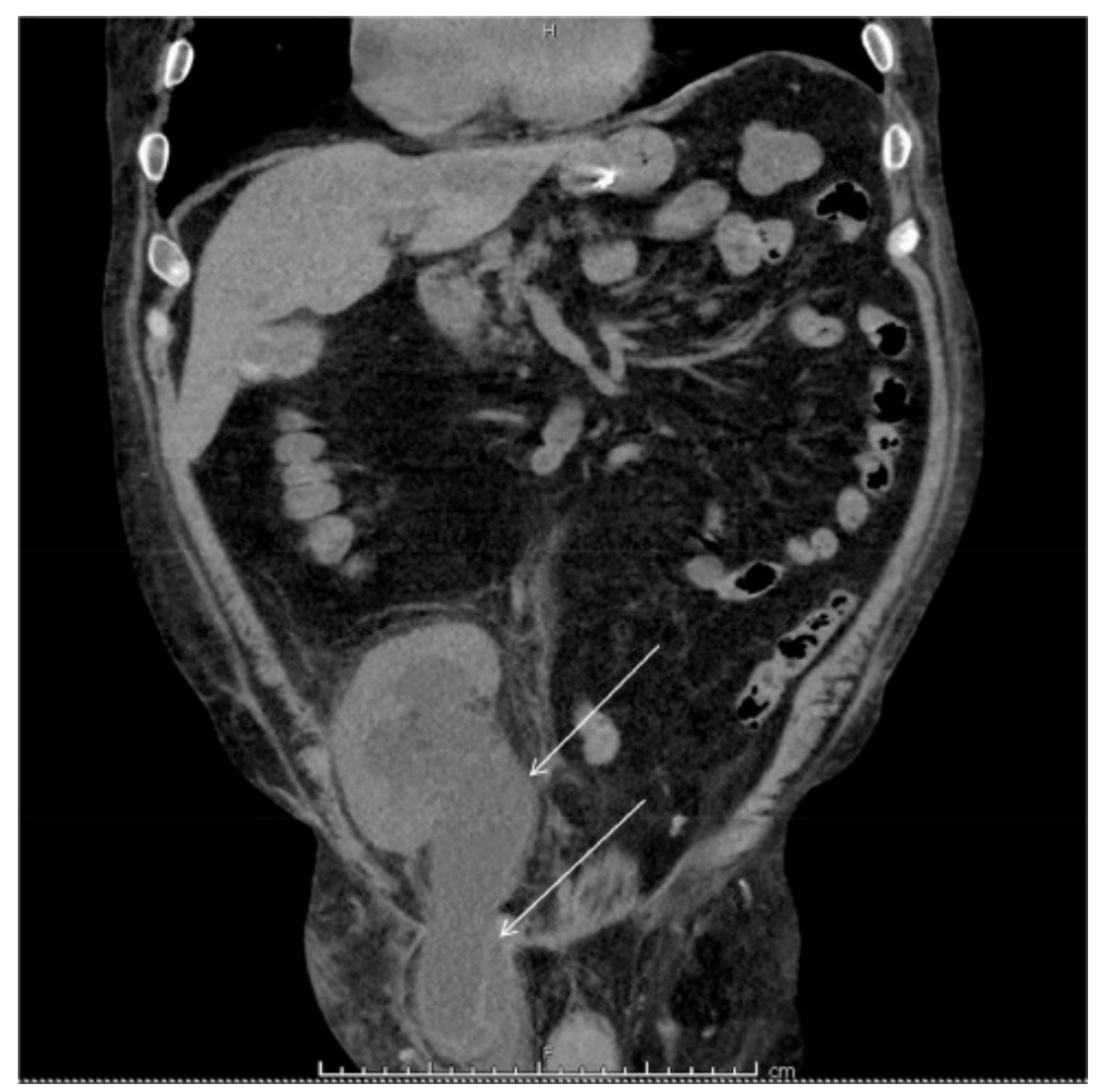

Image 1.

Non-Contrasted Abdominal and Pelvic CT scan (coronal plane) demonstrating obstruction of the right ureter of the transplanted kidney within a right inguinal hernia with resulting hydronephrosis and perinephric stranding (arrows) 


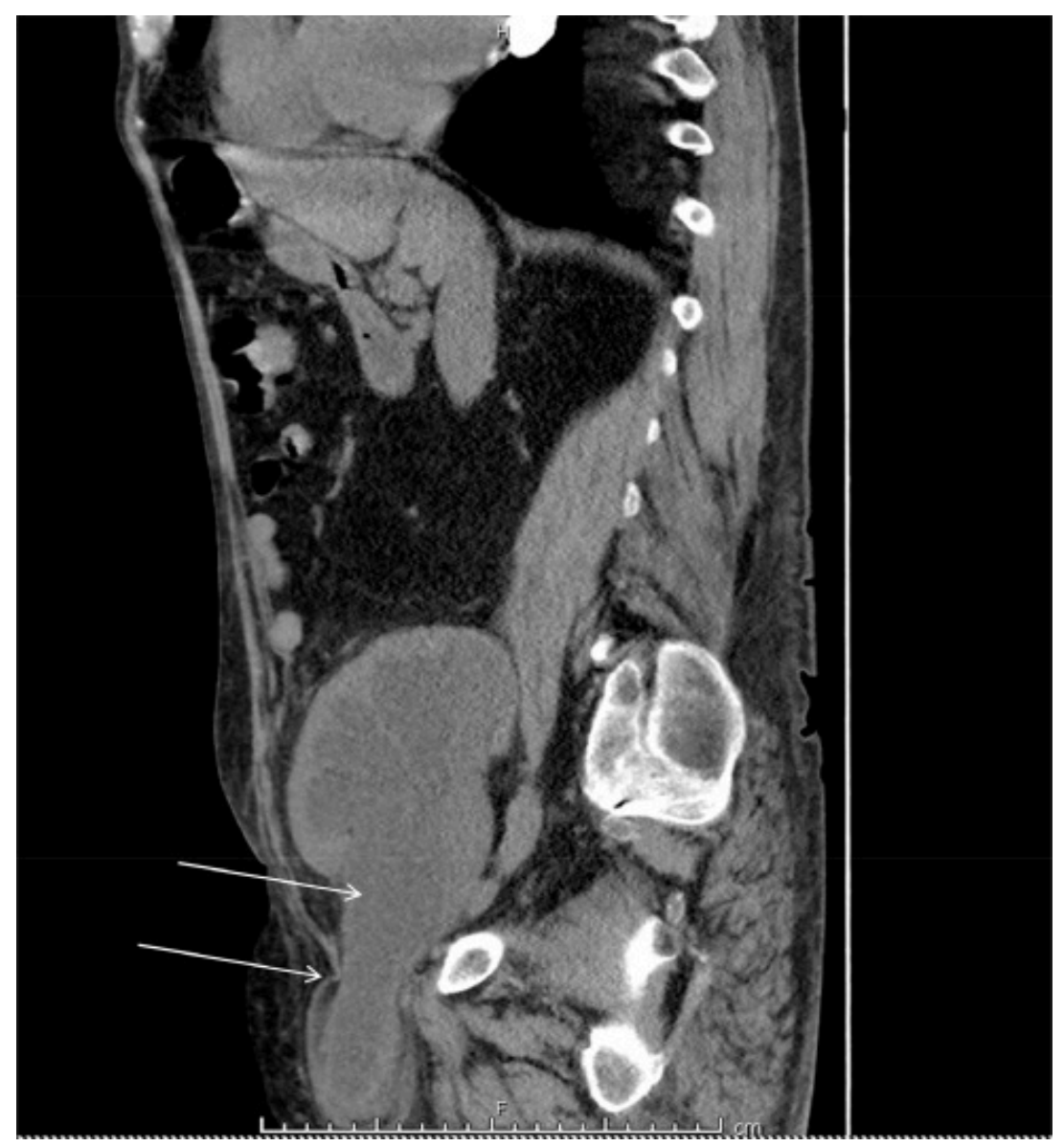

Image 2 .

Non-contrast Abdominal and Pelvic CT scan (sagittal plane) demonstrating obstruction of the right ureter of the transplanted kidney within a right inguinal hernia with resulting hydronephrosis and perinephric stranding (arrows). 


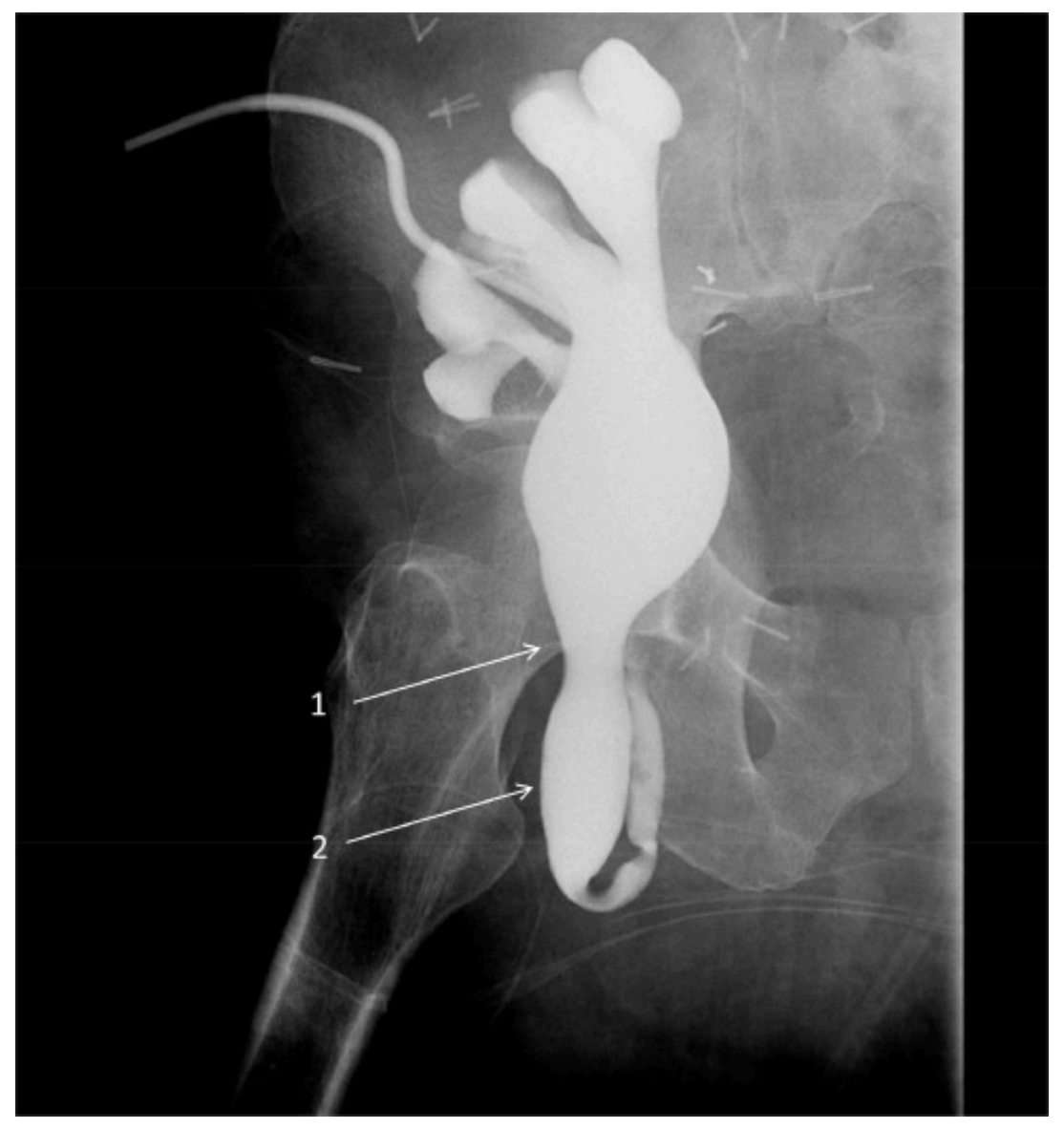

Image 3.

Nephrostogram Antegrade: Under fluoroscopic guidance, contrast was introduced thru the indwelling transplant percutaneous nephrostomy catheter. The image shows the nephrostomy catheter in the dilated transplant renal pelvis with marked dilatation of the calyces, pelvis, and ureter up to the level of the inguinal herniation (arrow 1). The mid ureter is inside the hernia (arrow 2) where a small amount of contrast is noted distally. There is no filling of the urinary bladder. 